\title{
Hilbert Space Representations of Lie Algebras
}

\author{
E. B. DAvies \\ Mathematical Institute Oxford, England \\ Received May 18, 1971
}

\begin{abstract}
We describe a new approach to the general theory of unitary representations of Lie groups which makes use of the Gelfand-Segal construction directly on the universal enveloping algebra of any Lie algebra. The crucial observation is that Nelson's theory of analytic vectors allows the characterisation of certain states on the universal enveloping algebra such that the corresponding representations of the universal enveloping algebra are the infinitesimal part of unitary representations of the associated simply connected Lie group. In the first section of the paper we show that with the aid of Choquet's theory of representing measures one can derive a simple new approach to integral decomposition theory along these lines.

In the second section of the paper we use these methods to study the irreducible unitary representations of general semi-simple Lie groups. We give a simple proof that the $K$-finite vectors studied by Harish-Chandra [5] are all analytic vectors. We also give new proofs of some of Godement's results [2] characterising spherical functions of height one, at least for unitary representations. Compared with [2] our method has the possible advantage of obtaining the characterisations by infinitesimal methods instead of using an indirect argument involving functions on the group. We point out that while being purely algebraic in nature, this approach makes almost no use of the deep and difficult theorems of HarishChandra concerning the universal enveloping algebra [5].

Our work is done in very much the same spirit as that of Power's recent paper [8]. The main difference is that by concentrating on a more special class of positive states we are able to carry the analysis very much further without difficulty.
\end{abstract}

\section{§ 1. Representations of the Universal Enveloping Algebra}

Let $G$ be a real simply connected Lie group with Lie algebra $\mathscr{G}$ and let $\mathscr{U}(\mathscr{G})$ denoted the complex universal enveloping algebra of $\mathscr{G}$. An involution is defined on $\mathscr{U}(\mathscr{G})$ by supposing that $X^{*}=-X$ for all $X \in \mathscr{G}$.

If $\pi$ is a unitary representation of $G$ on a Hilbert space $\mathscr{H}$, we denote by $\mathscr{H}^{\infty}$ and $\mathscr{H}^{\omega}$ the spaces of $C^{\infty}$ - and analytic vectors respectively for $\pi$. It is known [6] that both of these are always dense subspaces of $\mathscr{H}$. There is a representation $\partial \pi$ of $\mathscr{G}$ by operators on $\mathscr{H}^{\infty}$ given by

$$
\partial \pi(X) \xi=\lim _{t \rightarrow 0} t^{-1}\{\pi(\exp t X) \xi-\xi\}
$$

and this has a natural extension to a representation $\partial \pi$ of $\mathscr{U}(\mathscr{G})$. The extension is a ${ }^{*}$-representation in the sense that for all $X \in \mathscr{U}(\mathscr{G})$

$$
\partial \pi\left(X^{*}\right) \subseteq \partial \pi(X)^{*} .
$$


In terms of any basis $X_{1}, \ldots, X_{d}$ of $\mathscr{G}$ the analytic vectors of $\pi$ are determined by the following criterion, [6].

Proposition 1.1. A vector $\xi \in \mathscr{H}^{\infty}$ is an analytic vector for $\pi$ if and only if there are constants, $a, s>0$ such that

$$
\left\|\partial \pi\left(X_{i(1)} \ldots X_{i(n)}\right) \xi\right\| \leqq a n ! s^{-n}
$$

for all functions $i$ such that $1 \leqq i(r) \leqq d$ for all $1 \leqq r \leqq n$.

It is most important that this estimate can be deduced from an apparently weaker one involving the element $\Delta=X_{1}^{2}+\cdots+X_{d}^{2} \in \mathscr{U}(\mathscr{G})$, $[3,6]$.

Proposition 1.2. Let $\sigma$ be $a *$-representation of $\mathscr{U}(\mathscr{G})$ on a dense invariant domain $D$ in a Hilbert space $\mathscr{H}$. If $\xi \in D$ and there is a constant $M$ such that

$$
\left\|\sigma\left\{(1-\Delta)^{n}\right\} \xi\right\| \leqq M^{n}(2 n) !
$$

then there exists a constant s depending on $M$ but not on $\xi$ or $\sigma$ such that

$$
\left\|\sigma\left(X_{i(1)} \ldots X_{i(m)}\right) \xi\right\| \leqq \mathrm{s}^{-m} m ! .
$$

This proposition may be used in conjunction with the following sufficient condition for a $*$-representation of $\mathscr{U}(\mathscr{G})$ to be associated with a unitary representation of the Lie group $G,[6]$.

Proposition 1.3. Let $\sigma$ be $a^{*}$-representation of $\mathscr{U}(\mathscr{G})$ on a dense invariant domain $D$ in a Hilbert space $\mathscr{H}$ and let $s>0$ be a constant such that for all $\xi \in D$ there exists a constant $a_{\xi}$ such that

$$
\left\|\sigma\left(X_{i(1)} \ldots X_{i(n)}\right) \xi\right\| \leqq a_{\xi} n ! s^{-n} .
$$

Then there exists a unique unitary representation $\pi$ of $G$ on $\mathscr{H}$ such that $D \leqq \mathscr{H}^{\omega}$ and $\sigma$ is the restriction of $\partial \pi$ to $D$.

$$
\begin{aligned}
& \text { If } s_{1}^{2}+\cdots+s_{d}^{2} \leqq s \text { and } \xi \in D \text { then } \\
& \pi\left\{\exp \left(s_{1} X_{1}+\cdots+s_{d} X_{d}\right)\right\} \xi=\sum_{n} \sum_{i} \frac{1}{n !} \sigma\left(X_{i(1)} \ldots X_{i(n)}\right) \xi s_{i(1)} \ldots s_{i(n)}
\end{aligned}
$$

where the R.H.S. converges absolutely.

From these estimates it follows quickly that $\partial \pi(1-\Delta)$ is essentially self-adjoint on $\mathscr{H}^{\infty}$ and that $\mathscr{H}^{\omega}$ is precisely the set of analytic vectors for the square root of $\partial \pi(1-\Delta)^{-}$, [3].

We come now to the study of states on the universal enveloping algebra. A functional $\phi: \mathscr{U}(\mathscr{G}) \rightarrow \mathbb{C}$ is called a state if $\phi\left(X^{*} X\right) \geqq 0$ for all $X \in \mathscr{U}(\mathscr{G})$. This implies in particular that $\phi\left(X^{*}\right)=\overline{\phi(X)}$ for all $X \in \mathscr{U}(\mathscr{G})$. The set of all states forms a cone which defines a partial ordering on the linear space of all self-adjoint linear functionals on $\mathscr{U}(\mathscr{G})$. Those states which give rise to extreme rays of the cone are called pure states. By the 
Gelfand-Segal construction [1], corresponding to any state $\phi$ on $\mathscr{U}(\mathscr{G})$ there exists a Hilbert space $\mathscr{H}_{\phi}$, a ${ }^{*}$-representation $\sigma$ of $\mathscr{U}(\mathscr{G})$ by unbounded operators on a dense invariant subspace $D$ of $\mathscr{H}_{\phi}$ and a vector $\xi \in D$ such that for all $X \in \mathscr{U}(\mathscr{G})$

and $D=\sigma\{\mathscr{U}(\mathscr{G})\} \xi$.

$$
\langle\sigma(X) \xi, \xi\rangle=\phi(X)
$$

The question now arises whether the representation $\sigma$ can be exponentiated.

Theorem 1.4. Let $\phi$ be a state such that for some constant $M$ and all integers $n$

$$
\phi\left\{(1-\Delta)^{2 n}\right\} \leqq M^{2 n}(2 n) !^{2}
$$

Then there exists a unique unitary representation $\pi_{\phi}$ of $G$ on $\mathscr{H}_{\phi}$ such that $D \leqq \mathscr{H}_{\phi}^{\omega}$ and $\sigma$ is the restriction of $\partial \pi_{\phi}$ to $D$.

Proof. The given estimate may be written as

$$
\left\|\sigma(1-\Delta)^{n} \xi\right\| \leqq M^{n}(2 n) \text { ! }
$$

from which, by Proposition 1.2, we deduce an estimate of the form

$$
\left\|\sigma\left(X_{i(1)} \ldots X_{i(m)}\right) \xi\right\| \leqq s^{m} m ! .
$$

If $\eta \in D$ then $\eta=\sigma(X) \xi$ for some $X \in \mathscr{U}(\mathscr{G})$ and expanding $X$ as a polynomial in $X_{1}, \ldots, X_{\alpha}$ we obtain an estimate

$$
\left\|\sigma\left(X_{i(1)} \ldots X_{i(m)}\right) \eta\right\| \leqq a_{\eta} t^{m} m !
$$

where $t$ is any constant greater than $s$. The result then follows by Proposition 1.3.

We call a state satisfying an estimate of the above type an analytic state.

Theorem 1.5. If $\phi$ is an analytic state on $\mathscr{U}(\mathscr{G})$ then $\{\psi: 0 \leqq \psi \leqq \phi\}$ is order isomorphic to

$$
\left\{A \in \mathscr{L}\left(\mathscr{H}_{\phi}\right): 0 \leqq A \leqq 1 \text { and } A \pi_{\phi}(g)=\pi_{\phi}(g) A \text { for all } g \in G\right\} \text {. }
$$

In particular $\phi$ is a pure state if and only if $\pi_{\phi}$ is an irreducible unitary representation.

Proof. If $A$ is an operator of the stated type then $A$ leaves the subspace $\mathscr{H}^{\infty}$ invariant and $A \partial \pi(X)=\partial \pi(X) A$ for all $X \in \mathscr{U}(\mathscr{G})$. Therefore if $\psi$ is defined by

$\psi$ is a state and

$$
\psi(X)=\langle A \partial \pi(X) \xi, \xi\rangle
$$

$$
\begin{aligned}
\psi\left(X^{*} X\right) & =\langle A \partial \pi(X) \xi, \partial \pi(X) \xi\rangle \\
& \leqq\langle\partial \pi(X) \xi, \partial \pi(X) \xi\rangle \\
& =\phi\left(X^{*} X\right)
\end{aligned}
$$


Conversely given any $\psi$ of this type there exists by the Gelfand-Segal construction a bounded operator $A$ on $\mathscr{H}$ such that $0 \leqq A \leqq 1$ and

$$
\psi\left(Y^{*} X\right)=\langle A(\partial \pi X) \xi,(\partial \pi Y) \xi\rangle
$$

for all $X, Y \in \mathscr{U}(\mathscr{G})$. It follows that for all $X \in \mathscr{U}(\mathscr{G})$ and all $\eta, \zeta \in D$

$$
\left\langle(\partial \pi X) \eta, A^{*} \zeta\right\rangle=\left\langle A \eta,(\partial \pi X)^{*} \zeta\right\rangle .
$$

The required result now follows by exponentiating, using the fact that $D$ consists entirely of analytic vectors.

The following result allows us to derive the whole of integral decomposition theory for unitary representations of Lie groups from Choquet's theory of representing measures on compact convex sets [7]. The advantage of this over the usual approach [1] is that it brings the integral and infinitesimal aspects of Lie group theory into a much closer relationship.

Theorem 1.6. For any constant $M>0$ the set $K$ of states on $\mathscr{U}(\mathscr{G})$ such that

$$
\sum_{n=0}^{\infty}(2 n) !^{-2} M^{-2 n} \phi\left\{(1-\Delta)^{2 n}\right\} \leqq 1
$$

is a metrisable compact convex set and a cap for the cone of all states on $\mathscr{U}(\mathscr{G})$. Every state in $K$ can be represented as the barycentre of a probability measure concentrated on the set of pure states in $K$.

Proof. The set $K$ is clearly a convex cap and it is metrisable in the weak topology since $\mathscr{U}(\mathscr{G})$ has a countable basis. We have only now to prove that it is compact, since the last assertion then follows from [7].

By Proposition 1.2 there exists a constant $s$ such that for all $\phi \in K$

Therefore

$$
\left\|\sigma_{\phi}\left(X_{i(1)} \ldots X_{i(m)}\right) \xi\right\| \leqq s^{m} m ! .
$$

$$
\begin{aligned}
\left|\phi\left(X_{i(1)} \ldots X_{i(m)}\right)\right| & =\left|\left\langle\sigma_{\phi}\left(X_{i(1)} \ldots X_{i(m)}\right) \xi, \xi\right\rangle\right| \\
& \leqq s^{m} m ! .
\end{aligned}
$$

Since the elements $X_{i(1)} \ldots X_{i(m)}$ generate $\mathscr{U}(\mathscr{G})$, for every $X \in \mathscr{U}(\mathscr{G})$ there exists a constant $k_{X}<\infty$ such that for all $\phi \in K,|\phi(X)| \leqq k_{X}$. The compactness of $K$ is an immediate consequence.

One is not interested only in simply connected Lie groups, and the following criterion enables one to deal with more general situations.

Theorem 1.7. Let $G$ be a simply connected Lie group and $Z$ a discrete subgroup of the centre of $G$. For any $M>0$ let $K_{Z}$ be the set of states $\phi$ on $\mathscr{U}(\mathscr{G})$ such that

$$
\sum_{n=0}^{\infty}(2 n) !^{-2} M^{-2 n} \phi\left\{(1-\Delta)^{2 n}\right\} \leqq 1
$$


and $\pi(g)=1$ for all $g \in Z$, where $\pi$ is the unitary representation constructed from $\phi$. Then $K_{Z}$ is a weakly closed face of the compact convex set $K$.

Proof. If $0 \leqq \psi \leqq \phi \in K_{Z}$ then the Gelfand-Segal construction and Theorem 1.5 allow us to identify $\pi_{\psi}$ with a subrepresentation of $\pi_{\phi}$, from which it is immediate that $\pi_{\phi}(g)=1$ for all $g \in Z$, and hence $\psi \in K_{Z}$. This and similar calculations prove that $K_{Z}$ is a face and it remains to prove that it is weakly closed. Suppose $\phi_{n} \in K_{Z}$ and $\phi_{n} \rightarrow \phi_{0} \in K$. Each of these states defines via Theorem 1.4 a positive definite analytic function on $G$, say $f_{n}(g)=\left\langle\pi_{n}(g) \xi_{n}, \xi_{n}\right\rangle$, and by Proposition 1.3 and Theorem 1.4 it is clear that $f_{n}(g) \rightarrow f_{0}(g)$ for all $g$ in the neighbourhood

$$
N(e)=\left\{\exp \left(s_{1} X_{1}+\cdots+s_{d} X_{d}\right): s_{1}^{2}+\cdots+s_{d}^{2}<s^{2}\right\}
$$

of the identity in $G$. By the general theory of positive definite functions it now follows that $f_{n}(g) \rightarrow f_{0}(g)$ for all $g \in G$ and so $f_{0}(g)=1$ for all $g \in Z$. But if $\left\langle\pi_{0}(g) \xi, \xi\right\rangle=1$ then $\pi_{0}(g) \xi=\xi$, by spectral theory, so $\phi_{0} \in K_{Z}$.

\section{$\S$ 2. Spherical Functions on Semi-simple Matrix Groups}

In this section we use the above results to provide a new derivation of some of Godement's results on spherical functions for unitary representations of semi-simple groups. More general results can be found in $[2,5]$ but our approach avoids all technical problems, and derives the infinitesimal results directly, rather than obtaining them from the integral versions as Godement does.

We let $G$ be a real semi-simple, simply connected group with Lie algebra $\mathscr{G}$. Let $\mathscr{G}=\mathscr{K}+\mathscr{P}$ be a Cartan decomposition of $\mathscr{G}$ and $X_{1}, \ldots, X_{e}$ a basis for $\mathscr{K}, X_{e+1}, \ldots, X_{d}$ as basis for $\mathscr{P}$ with respect to which the Killing form $B: \mathscr{G} \times \mathscr{G} \rightarrow \mathbb{R}$ is given by

$$
B\left(X_{i}, X_{j}\right)=\left\{\begin{array}{c}
0, i \neq j \\
-1, i=j \leqq e \\
+1, i=j>e .
\end{array}\right.
$$

If $\mathscr{U}(\mathscr{G})$ is the complex universal enveloping algebra of $\mathscr{G}$ then the subalgebra generated by $\mathscr{K}$ is isomorphic with $\mathscr{U}(\mathscr{K})$. The element $C_{1}=-X_{1}^{2}-\cdots-X_{e}^{2}+X_{e+1}^{2}+\cdots+X_{d}^{2}$ lies in the centre $Z(\mathscr{G})$ of $\mathscr{U}(\mathscr{G})$ and the element $C_{2}=-X_{1}^{2} \ldots-X_{e}^{2}$ lies in the centre $Z(\mathscr{K})$ of $\mathscr{U}(\mathscr{K})$. Moreover in our earlier notation $\Delta=C_{1}-2 C_{2}$.

If $K$ is the Lie subgroup of $G$ associated with $\mathscr{K}$ then $K$ need not be compact if the centre $Z$ of $G$ is too large. However we may write $K=K_{1} \times K_{2}$ where $K_{1}$ is a compact group, $K_{2}$ is a vector group and $\bar{K}=K / Z \cap K_{2}$ is compact. We observe that for the adjoint representation on $\mathscr{U}(\mathscr{G}), K$ may be factored through $\bar{K}$ so the image is a compact group. 
Lemma 2.1. If $\pi$ is an irreducible unitary representation of $G$ on a Hilbert space $\mathscr{H}$ then $\pi \mid K$ is a direct sum of irreducible representations.

Proof. Since $\pi$ is irreducible $\pi \mid Z$ is given by a character. If $\chi: K$ $\rightarrow\{z:|z|=1\}$ is a character such that $\chi(g)=\pi(g)$ for all $g \in Z \cap K_{2}$ then the representation $\pi^{\prime}(k)=\pi(k) \cdot \chi(k)^{-1}$ of $K$ can be factored through $\bar{K}$. The result follows from standard theorems about representations of compact groups.

We define the positive cone of $\mathscr{U}(\mathscr{G})$ to be the weakly closed cone generated by $\left\{X^{*} X: X \in \mathscr{U}(\mathscr{G})\right\}$ and define $\mathscr{U}_{0}(\mathscr{G})$ to be $\{X \in \mathscr{U}(\mathscr{G})$ $: \operatorname{ad} k(X)=X$ for all $k \in K\} . \mathscr{U}_{0}(\mathscr{G})$ is a ${ }^{*}$-subalgebra of $\mathscr{U}(\mathscr{G})$ containing $Z(\mathscr{K})$ and $Z(\mathscr{G})$, and we define its positive cone to be its intersection with the + ve cone of $\mathscr{U}(\mathscr{G})$. Since $K$ has an action on the semi-graded algebra $\mathscr{U}(\mathscr{G})$ there is an averaging map' ${ }^{4}$ on $\mathscr{U}(\mathscr{G})$ defined by

$$
X^{\natural}=\int_{\overline{\mathrm{K}}} \operatorname{ad} \bar{k}(X) d \bar{k} .
$$

This is a positive linear map of $\mathscr{U}(\mathscr{G})$ onto $\mathscr{U}_{0}(\mathscr{G})$, and in particular satisfies $\Delta^{\natural}=\Delta$. We finally define $\mathscr{M}$ to be the set of all positive homomorphisms from $\mathscr{U}_{0}(\mathscr{G})$ to $\mathbb{C}$ and give $\mathscr{M}$ the weak topology. It is clear that $\mathscr{U}_{0}(\mathscr{G})$ commutes with $\mathscr{U}(\mathscr{K})$ and we denote by $\mathscr{B}$ the algebra generated by these.

For each $X \in \mathscr{U}(\mathscr{G})$ we denote by $\tilde{X}$ the associated left invariant differential operator on $C^{\infty}(G)$ so that if $\pi$ is a representation of $G$ and $\xi, \eta \in \mathscr{H}^{\infty}$,

$$
\tilde{X}\langle\pi(g) \xi, \eta\rangle=\langle\pi(g) \partial \pi(X) \xi, \eta\rangle .
$$

The elements of $\mathscr{U}_{0}(\mathscr{G})$ then correspond to the differential operators on $G$ which are left invariant with respect to $G$ and right invariant with respect to $K$.

If $\pi$ is any representation of $G$ and $\sigma$ is an irreducible representation of $K$ we denote by $\mathscr{H}_{\sigma}$ the closed subspace of $\mathscr{H}$ consisting of vectors which transform like $\sigma$ under $\pi \mid K$. Clearly $\mathscr{H}=\sum_{\sigma \in K} \mathscr{H}_{\sigma}$.

Lemma 2.2. If $\pi$ is an irreducible unitary representation of $G$ on $\mathscr{H}$ and $\sigma \in \hat{K}$ then $\mathscr{H}_{\sigma} \subseteq \mathscr{H}^{\omega}$ and $\partial \pi$ maps $\mathscr{B}$ onto an irreducible $*_{\text {-algebra of }}$ bounded operators on $\mathscr{H}_{\sigma}$.

Proof. One first observes that $\partial \pi\left(C_{1}\right)$ is a scalar operator, by [9]. Also $\partial \pi\left(C_{2}\right)$ leaves $\mathscr{H}_{\sigma}$ invariant and is a scalar operator on $\mathscr{H}_{\sigma}$. Therefore $\mathscr{H}_{\sigma}$ is an eigenspace for $\partial \pi(\Delta)=\partial \pi\left(C_{1}-2 C_{2}\right)$. The fact that $\mathscr{H}_{\sigma} \subseteq \mathscr{H}^{\omega}$ now follows from Propositions 1.1 and 1.2. It is easy to see from their definitions that $\partial \pi(X)$ leaves $\mathscr{H}_{\sigma}$ invariant for all $X \in \mathscr{U}(\mathscr{K})$ and all $X \in \mathscr{U}_{0}(\mathscr{G})$, so $\partial \pi$ maps $\mathscr{B}$ onto a ${ }^{*}$-algebra of operators on $\mathscr{H}_{\sigma}$. Since 
$\mathscr{H}_{\sigma}=\mathscr{H}_{\sigma} \cap \mathscr{H}^{\infty}$ the norm topology of $\mathscr{H}_{\sigma}$ must coincide with its $C^{\infty}$ Frechet space topology defined in [4], the operators $\partial \pi(X) \mid \mathscr{H}_{\sigma}$ must be continuous and so bounded for all $X \in \mathscr{B}$.

We may write $\mathscr{H}_{\sigma}=\mathscr{H}_{1} \otimes \mathscr{H}_{2}$ and $\partial \pi \mid \mathscr{B}=\partial \pi_{1} \otimes \partial \pi_{2}$ where $\partial \pi_{1}$ maps $\mathscr{U}(\mathscr{K})$ onto the algebra of all operators on the finite-dimensional space $\mathscr{H}_{1}$ and $\partial \pi_{2}$ maps $\mathscr{U}_{0}(\mathscr{G})$ into $\mathscr{L}\left(\mathscr{H}_{2}\right)$. Let $\xi_{1}, \ldots, \xi_{n}$ be an orthonormal basis for $\mathscr{H}_{1}$ and let $\xi, \eta \in \mathscr{H}_{2}$. Since $\pi$ is irreducible and $\mathscr{U}(\mathscr{G}) \supseteqq \mathscr{U}(\mathscr{K})$ there exists $X \in \mathscr{U}(\mathscr{G})$ such that

$$
\left\langle\partial \pi(X) \xi_{i} \otimes \xi, \xi_{j} \otimes \eta\right\rangle=\delta_{i j}
$$

for $1 \leqq i, j \leqq n$. Then letting $\bar{\sigma}$ be a rep of $\bar{K}$ projectively equivalent to the rep $\sigma$ of $K$, it follows that

$$
\begin{aligned}
\left\langle\partial \pi_{2}\left(X^{\natural}\right) \xi, \eta\right\rangle & =\left\langle\partial \pi\left(X^{\natural}\right) \xi_{1} \otimes \xi, \xi_{1} \otimes \eta\right\rangle \\
& =\int_{\bar{K}}\left\langle\sigma\left(\bar{k}^{-1}\right) \partial \pi(X) \sigma(\bar{k}) \xi_{1} \otimes \xi, \xi_{1} \otimes \eta\right\rangle d \bar{k} \\
& =\sum_{i, j=1}^{n} \int_{\bar{K}}\left\langle\sigma(\bar{k}) \xi_{1}, \xi_{i}\right\rangle\left\langle\sigma(\bar{k}) \xi_{1}, \xi_{j}\right\rangle^{-}\left\langle\partial \pi(X) \xi_{i} \otimes \xi, \xi_{j} \otimes \eta\right\rangle d \bar{k} \\
& =n \sum_{i=1}^{n} \int_{\bar{K}}\left|\left\langle\sigma(\bar{k}) \xi_{1}, \xi_{i}\right\rangle\right|^{2} d \bar{k} \\
& >0 .
\end{aligned}
$$

This is enough to prove that the algebra $\partial \pi_{2}\left(\mathscr{U}_{0}(\mathscr{G})\right.$ acts irreducibly on $\mathscr{H}_{2}$.

If $\pi$ is an irreducible unitary representation of $G$ and $\sigma$ is an irreducible representation of $K$ which occurs with multiplicity one in $\pi \mid K$, and if $\xi_{1}, \ldots, \xi_{n}$ is a basis for $\mathscr{H}_{\sigma}$ then the function

$$
f(g)=\sum_{i=1}^{n}\left\langle\pi(g) \xi_{i}, \xi_{i}\right\rangle
$$

is called a positive definite spherical function of height one associated with $\pi, \sigma,[2]$. It is clear that such a function is analytic and satisfies $f\left(\mathrm{kgk}^{-1}\right)$ $=f(g)$ for all $k \in K$.

Theorem 2.3. (Godement). The formula

$$
X(m)=(\tilde{X} f)(e) \cdot \operatorname{dim} \sigma
$$

valid for all $X \in \mathscr{U}_{0}(\mathscr{G})$, defines a one-one correspondence between the set of all positive definite spherical functions $f$ of height one, and the set of all $m \in \mathscr{M}$. The space $\mathscr{M}$ is locally compact. The positive definite spherical functions of height one are precisely those positive definite $C^{\infty}$-functions on $G$ which are eigenfunctions of all the differential operators $\tilde{X}$ such that $X \in \mathscr{U}_{0}(\mathscr{G})$, and satisfy $f\left(\mathrm{kgk}^{-1}\right)=f(g)$ for all $k \in K$ and $g \in G$. 
Proof. Given $\pi$, define $\phi: \mathscr{U}(\mathscr{G}) \rightarrow \mathbb{C}$ by

$$
\begin{aligned}
\phi(X) & =\sum_{i=1}^{n}\left\langle\partial \pi(X) \xi_{i}, \xi_{i}\right\rangle \\
& =\operatorname{tr}\left[P_{\sigma} \partial \pi(X) P_{\sigma}\right]
\end{aligned}
$$

where $P_{\sigma}$ is the projection on the subspace $\mathscr{H}_{\sigma}$. It is clear that $\phi$ is positive definite and that $\phi((\operatorname{ad} k) X)=\phi(X)$ for all $X \in \mathscr{U}(\mathscr{G})$ and $k \in K$. Therefore $\phi(X)=\phi\left(X^{\natural}\right)$. If $\sigma$ is of multiplicity one in $\pi \mid K$ then $\partial \pi(X)$ is a scalar operator on $\mathscr{H}_{\sigma}$ for all $X \in \mathscr{U}_{0}(\mathscr{G})$ so $\phi \mid \mathscr{U}_{0}(\mathscr{G})$ is a character $m \in \mathscr{M}$. It is clear that if $f$ is the spherical function on $G$ associated with $\pi, \sigma$ then for all $X \in \mathscr{U}(\mathscr{G})$

$$
\begin{aligned}
(X f)(e) & =\sum_{i=1}^{n}\left\langle\pi(e) \partial \pi(X) \xi_{i}, \xi_{i}\right\rangle=\phi(X)=\phi\left(X^{\natural}\right) \\
& =(\operatorname{dim} \sigma) X^{\natural}(m) .
\end{aligned}
$$

Since $f$ is analytic it is determined by its derivatives at the origin and hence by $\sigma$ and $m$. However $\mathscr{U}_{0}(\mathscr{G}) \geqq Z(\mathscr{K})$ and since $\operatorname{ad} K$ is compact $\sigma$ is determined by the associated character on $Z(\mathscr{K})$, which is of course just the restriction of $m$ to $Z(\mathscr{K})$. Therefore $m$ determines $\sigma$ and so $f$.

The only non-trivial part of the argument consists of showing that every $m \in \mathscr{M}$ arises from some spherical function. Let $m \in \mathscr{M}$ and define $\phi$ on $\mathscr{U}(\mathscr{G})$ by $\phi(X)=X^{\natural}(m)$, so that $\phi$ is a positive definite functional. Since $\phi\left(\Delta^{n}\right)=\left(\Delta^{n}\right)^{\natural}(m)=\{\Delta(m)\}^{n}$ it is clear that $\phi$ is an analytic state. Define

$$
\mathscr{J}=\left\{X \in \mathscr{U}_{0}(\mathscr{G}): \phi\left(X^{*} X\right)=0\right\}
$$

and $F$ as the set of positive functionals $\psi$ on $\mathscr{U}(\mathscr{G})$ such that $\psi\left(X^{*} X\right)=0$ for all $X \in \mathscr{J}$ and

$$
\sum_{n=0}^{\infty}(2 n) !^{-2}\{1-\Delta(m)\}^{-2 n} \phi\left\{(1-\Delta)^{2 n}\right\} \leqq 1 .
$$

Then $F$ is a non-empty weakly closed face of the set $K$ of analytic states defined in Theorem 1.6. Moreover if

then

$$
X \in \mathscr{U}_{0}(\mathscr{G}) \text { and } \phi(X)=\alpha
$$

so for all $\psi \in F$

$$
\phi\left((X-\alpha)^{*}(X-\alpha)\right)=|\phi(X)-\phi(\alpha)|^{2}=0
$$

$$
|\psi(X-\alpha)|^{2} \leqq \psi(1) \psi\left\{(X-\alpha)^{*}(X-\alpha)\right\}=0 .
$$

Therefore $\psi(X)=\psi(1) \phi(X)$ for all $X \in \mathscr{U}_{0}(\mathscr{G})$. Since $F$ is a closed face of $K$ there exists an extreme point $\psi$ of $F$, and this is also an extreme point of $K$. Let $\pi$ be the corresponding irreducible representation of $G$ with 
analytic cyclic vector $\xi$. Since $Z(\mathscr{K}) \subseteq \mathscr{U}_{0}(\mathscr{G}), \partial \pi(X) \xi=X(m) \xi$ for all $X \in Z(\mathscr{K})$. Since $Z(\mathscr{K})$ separates the irreducible representations of $\mathscr{K}$, $\xi \in \mathscr{H}_{\sigma}$ for some single irreducible representation $\sigma$ of $\mathscr{K}$.

If $X \in \mathscr{U}_{0}(\mathscr{G})$ then $\partial \pi(X) \xi \in \mathscr{H}_{\sigma}$ and

$$
\begin{aligned}
\|\partial \pi(X) \xi-m(X) \xi\|^{2} & =\psi\left((X-m(X))^{*}(X-m(X))\right) \\
& =0
\end{aligned}
$$

so $\partial \pi(X) \xi=m(X) \xi$ and in fact if $Y \in \mathscr{U}(\mathscr{K})$ and $\eta=\partial \pi(Y) \xi$

$$
\partial \pi(X) \eta=\partial \pi(X) \partial \pi(Y) \xi=m(X) \eta .
$$

Therefore the algebra of operators $\partial \pi(\mathscr{B})$ on $\mathscr{H}_{\sigma}$ is the same as $\partial \pi(\mathscr{U}(\mathscr{K}))$. By Lemma 2.2, $\mathscr{K}$ acts irreducibly on $\mathscr{H}_{\sigma}$ so $\sigma$ has multiplicity one in $\pi \mid K$. If $\xi_{1}, \ldots, \xi_{n}$ is a basis of $\mathscr{H}_{\sigma}$ and

$$
f(g)=\sum_{i=1}^{n}\left\langle\pi(g) \xi_{i}, \xi_{i}\right\rangle
$$

then $f(g)$ is a spherical function and for $X \in \mathscr{U}(\mathscr{G})$

$$
\begin{aligned}
(\tilde{X} f)(e) & =\sum_{i=1}^{n}\left\langle\partial \pi(X) \xi_{i}, \xi_{i}\right\rangle \\
& =\sum_{i=1}^{n}\left\langle\partial \pi\left(X^{\natural}\right) \xi_{i}, \xi_{i}\right\rangle \\
& =(\operatorname{dim} \sigma) X^{\natural}(m) .
\end{aligned}
$$

This shows that the map of the theorem has range equal to $\mathscr{M}$.

To prove that $\mathscr{M}$ is locally compact in the weak topology we define

$$
\mathscr{M}_{\alpha}=\{m \in \mathscr{M}: \Delta(m) \leqq \alpha\}
$$

for any $\alpha<\infty$. If $m \in \mathscr{M}_{a}$ and $\phi_{m}(X)=X^{\natural}(m)$ then $\phi_{m}$ is a positive state and $\phi_{m}\left(\Delta^{2 n}\right) \leqq \alpha^{2 n}$. By the proof of Theorem 1.6 there is a constant $\beta$, depending only on $\alpha$, such that if $m \in \mathscr{M}_{\alpha}$

This implies that

$$
\left|\phi_{m}\left(X_{i(1)} \ldots X_{i(n)}\right)\right| \leqq n ! \beta^{n} .
$$

$$
\left\{|m(X)|: m \in \mathscr{M}_{\alpha}\right\}
$$

is a bounded set for any $X \in \mathscr{U}_{0}(\mathscr{G})$, which proves that $\mathscr{M}_{\alpha}$ is compact. Since $\mathscr{M}_{\alpha} \subseteq \operatorname{int}\left(\mathscr{M}_{\alpha+1}\right)$ it follows that $\mathscr{M}$ is locally compact.

Finally we show that any $C^{\infty}$ function satisfying the conditions of the last statement of the theorem is a spherical function. If $\pi, \xi$ is the cyclic representation associated with $f$, then by [4], $\xi$ is a $C^{\infty}$-vector for $\pi$. If $\phi: \mathscr{U}(\mathscr{G}) \rightarrow \mathbb{C}$ is defined by $\phi(X)=\langle\partial \pi(X) \xi, \xi\rangle$ and if $\tilde{X} f=\lambda f$ for some 


$$
\begin{aligned}
X \in \mathscr{U}_{0}(\mathscr{G}) & \\
\left\langle\partial \pi(X) \xi-\lambda \xi, \pi\left(g^{-1}\right) \xi\right\rangle & =\langle\pi(g) \partial \pi(X) \xi, \xi\rangle-\lambda\langle\pi(g) \xi, \xi\rangle \\
& =(\tilde{X} f)(g)-\lambda f(g) \\
& =0 .
\end{aligned}
$$

As $\xi$ is a cyclic vector for $\pi, \partial \pi(X) \xi=\lambda \xi$. Therefore $\phi \mid \mathscr{U}_{0}(\mathscr{G})$ is a character. Moreover since

$$
\phi(X)=(\tilde{X} f)(e)
$$

and $f(g)=f\left(k g k^{-1}\right)$ for all $k \in K, \phi(X)=\phi(\operatorname{ad} k X)$. Therefore $\phi(X)$ $=\phi\left(X^{\natural}\right)$. It is immediate that $\phi$ is an analytic state, $\xi$ is an analytic vector, and $f$ is an analytic function, the spherical function corresponding to an element of $\mathscr{M}$.

\title{
References
}

1. Dixmier, J.: Les $C^{*}$-algèbres et leurs représentations, cahiers scientifiques, fasc xxix. Paris: Gauthiers-Villars 1964.

2. Godement, R.: A theory of spherical functions I. Trans. Amer. Math. Soc. 73, 496-556 (1952).

3. Goodman, R.: Analytic domination by fractional powers of a positive operator. J. Functional Anal. 3, 246-264 (1969).

4. - Analytic and entire vectors for representations of Lie groups. Trans. Amer. Math. Soc. 143, 55-76 (1969).

5. Harish-Chandra: Representations of a semi-simple Lie group on a Banach space, I. Trans. Amer. Math. Soc. 75, 185-243 (1953).

6. Nelson, E.: Analytic vectors. Ann. of Math. 70, 572-615 (1959).

7. Phelps, R.R.: Lectures on Choquet's theorem. Princeton, New Jersey: van Nostrand 1966.

8. Powers, R. T.: Self-adjoint algebras of unbounded operators. Commun. math. Phys. 21, 85-124 (1971).

9. Segal,I.E.: Hypermaximality of certain operators on Lie groups. Proc. Amer. Math. Soc. 3, 13-15 (1952).

\author{
E. B. Davies \\ Mathematical Institute \\ $24-29$ St. Giles \\ Oxford \\ England
}

